\title{
Enrichment and mutation detection of circulating tumor cells from blood samples
}

\author{
RUQIN KOU ${ }^{1}$, JIAN ZHAO $^{1}$, PRIYA GOGOI ${ }^{2}$, SHANNON CARSKADON $^{3}$, WILL CHOW $^{2}$, CLARA HWANG $^{4}$, \\ NALLASIVAM PALANISAMY ${ }^{3 *}$, CONRAD LEUNG $^{{ }^{*}}$ and YIXIN WANG ${ }^{2 *}$ \\ ${ }^{1}$ Research and Development, GENEWIZ, South Plainfield, NJ 08070; ${ }^{2}$ Celsee Diagnostics, Plymouth, MI 48170; \\ Departments of ${ }^{3}$ Urology, and ${ }^{4}$ Hematology and Oncology, Henry Ford Health System, Detroit, MI 48202, USA
}

Received September 29, 2017; Accepted March 23, 2018

DOI: $10.3892 /$ or.2018.6342

\begin{abstract}
The potential of circulating tumor cells (CTCs) in the diagnosis and prognosis of cancer patients has become increasingly attractive. However, molecular analysis of CTCs is hindered by low sensitivity and a high level of background leukocytes in CTC enrichment technologies. We have developed a novel protocol using a microfluidic device, which enriches and retrieves CTCs from blood samples. The principle of CTC capturing is that tumor cells are larger and less deformable than normal blood cells. To evaluate the potential of utilizing Celsee PREP100 in CTC molecular analysis, we prepared prostate cancer cell lines PC 3 and LNCaP, retrieved the captured cells and analyzed them using PCR amplicon sequencing. We were able to recover an average of $79 \%$ of 110-1,100 PC3 and 60-1,500 LNCaP cells, and detect the p.K139fs*3 deletion of the p53 gene in PC3 cells and p.T877A mutation of the androgen receptor gene in $\mathrm{LNCaP}$ cells. Next, we spiked these two types of cells into normal donor blood samples, captured the cells and analyzed them using PCR amplicon sequencing. The PC 3 and LNCaP cells were captured and retrieved with the ratio of captured CTCs to the background leukocytes reaching 1:1.5 for PC 3 and 1:2.9 for $\mathrm{LNCaP}$ cells. We further revealed that the p.K139fs*3 deletion and p.T877A mutation can be detected in the captured PC3 and LNCaP cells, respectively. We successfully validated this approach using clinical blood samples from patients with metastatic prostate cancer. Our results demonstrated a novel approach for CTC enrichment and illustrated the potential of CTC molecular characterization for diagnosis, prognosis and treatment selection of patients with metastatic malignancy.
\end{abstract}

Correspondence to: Dr Yixin Wang, Celsee Diagnostics, 46701 Commerce Center Drive, Plymouth, MI 48170, USA

E-mail: ywang@celsee.com

"Joint senior authorship

Key words: microfluidic chip, prostate cancer, CTCs, cell retrieval, sequencing, fluorescence in situ hybridization

\section{Introduction}

Circulating tumor cells (CTCs) have been identified in the blood and bone marrow of patients with breast, prostate and colon cancers (1-3) in as low as 1/100 million or 1 billion blood cells. Molecular characterization of CTCs may provide a greater understanding of the disease metastases, identify aggressive tumors and enable therapeutic selection and monitoring of the disease for patients undergoing treatment $(4,5)$. A variety of technologies have been developed to improve detection and capture of CTCs from peripheral blood, which include immune-magnetic bead separation using monoclonal antibodies targeting cell-surface antigens for positive or negative selection, cell sorting using flow cytometry, filtration-based size separation, density gradient centrifugation, microfluidic devices and fast-scan imaging (6-10). For example, CellSearch ${ }^{\mathrm{TM}}$ was the first CTC technology that demonstrated its clinical validity in predicting progression-free and overall survival of metastatic cancer patients based on CTC enumeration (3-6).

It is of great interest to go beyond cell enumeration and further characterize the CTCs by assessing clinically relevant molecular markers on or within CTCs to gain insight into the mechanisms of metastasis and best treatment modalities for patients $(1-3,11,12)$. For example, significant progress has been made in breast cancer, including effective hormonal therapy, chemotherapy and targeted therapies against estrogen receptor (ER) and HER-2. In prostate cancer, androgen receptor (AR) variant 7 has been implicated in predicting response to targeted therapies on AR. Established clinical, pathological features and biomarker status are routinely used to guide treatment options. It has become critically important to determine which patients are most likely to benefit from specific therapies. Detecting such molecular markers using a minimally-invasive blood test for CTCs has great potential in clinical practice to guide therapy choice for patients. However, despite advances in CTC technologies, the low frequency of CTCs in cancer patients and the extensive background leukocytes have limited the synergism of biomarkers and CTC technologies $(11,12)$.

We have developed a novel microfluidic device, Celsee PREP100 that uses a size and deformability-based capturing mechanism of CTCs (13). The microfluidic chip has a parallel network of fluidic channels which contain about 
56,000 capture chambers $(13,14)$. The chip fabrication begins with a silicon master device containing micro-features that make up a fluidic network (75- $\mu \mathrm{m}$ deep), leading to individual cell trapping chambers $(20 \times 25 \times 30 \mu \mathrm{m})$ with a pore size of 10x8 $\mu \mathrm{m}$. Each chamber ensures smaller blood cells such as red blood cells and most of the leukocytes escape while larger cancer cells get trapped and isolated in the chamber. The manufacturing process uses standard photo-lithography and deep reactive ion etching for micro-fabrication. From the master device, a soft elastomeric negative mold is created by pouring and curing against the silicon master. The final micro-substrate is created by hot embossing a plastic plate made of cyclic olefin polymer (COP) against the elastomeric negative mold. A thin plastic laminate containing pressure-sensitive adhesive is then laminated against the COP micro-substrate to create the final microfluidic chip. The chip is placed on the Celsee PREP100 device for CTC capturing.

Since the device captures cells using a label-free mechanism, it provides an improved sensitivity in capturing CTCs and an open platform for investigators to use a variety of antibodies to identify and characterize CTCs upon capturing $(13,14)$. In a previous study, we compared CTC enumeration between the Celsee system and the FDA-cleared CellSearch system using blood samples from patients with metastatic prostate cancer. CTC counts were significantly higher using the Celsee system (14). The captured CTCs could also be retrieved reproducibly using a back-flow procedure from the microfluidic chip for further nucleic acid extraction and molecular analysis. In the present study, we report the development of a novel protocol for capturing cells using blood sample retrieval and analysis of the cells using PCR amplicon sequencing. Using this method, we evaluated the potential of applying Celsee PREP100 in CTC molecular analysis by analyzing p.K139fs*3 of TP53 and p.T877A of AR in captured prostate cancer cell lines PC3 and LNCaP and in captured spiked-in cells in normal donor blood. The method was also tested successfully in clinical blood samples from 11 patients with metastatic prostate cancer successfully. Our results demonstrated the potential of utilizing CTCs for diagnosis, prognosis and treatment selection of patients with metastatic malignancy.

\section{Materials and methods}

Materials. Prostate cancer cell lines PC3 and LNCaP were purchased from the American Type Culture Collection (ATCC; Manassas, VA, USA) and cultured in Dulbecco's modified Eagle's medium (DMEM) containing 5\% fetal bovine serum (FBS) in a humidified incubator supplemented with $5 \% \mathrm{CO}_{2}$. Upon confluency, cells were digested with $0.5 \%$ trypsin and passaged at a 1:4 ratio. Some cells were resuspended in culture medium, counted and used for experiments. Blood samples were obtained from healthy donors. Clinical blood samples from patients with metastatic prostate cancer were obtained at Henry Ford Health System (Detroit, MI, USA). The present study was approved by the Institutional Review Board (IRB) of the Henry Ford Health System and written consent was obtained from all patients. Following informed consent, blood samples from patients were acquired in $10 \mathrm{ml}$ BCT Cell-Free DNA tubes (Streck, La Vista, NE, USA) or EDTA-coated Vacutainer ${ }^{\circledR}$ tubes (BD Biosciences, Franklin Lakes, NJ, USA).
Removal of CD45-positive cells from blood samples and CTC enrichment and staining. To test CTC enrichment efficiency using the Celsee PREP100 instrument, 250 PC3 and $50 \mathrm{LNCaP}$ cells were spiked into $4 \mathrm{ml}$ of blood from healthy donors. CD45-positive cells were removed using the RosetteSep Human CD45 Depletion Cocktail (Stemcell Technologies, Inc., Cambridge, MA, USA) following the manufacturer's protocol. The upper layer of plasma cells was collected and added into the inlet funnel of the Celsee PREP100. Cells were enriched in the microfluidic chip and stained for cytokeratins, CD45 and nuclei using anti-Pan cytokeratin antibody (1:100 dilution; cat. no. 914204), anti-CD45 antibody (1:100 dilution; cat. no. 368515; both antibodies were from BioLegend, Inc., San Diego, CA, USA) and DAPI, using Celsee PREP100 CTC Immunochemistry kit (Celsee Diagnostics, Plymouth, MI, USA). Cells enriched on the slides were counted using the Celsee Analyzer (14). Cytokeratins and DAPI-positive and CD45-negative cells were counted as CTC cells. The enrichment efficiency was calculated as the percentage of the enriched cells of the total spiked-in cells.

Cell retrieval using the Celsee PREP100 instrument. A different amount of PC3 and LNCaP cells were spiked into the priming buffer and retrieved in $2 \mathrm{ml}$ phosphate-buffered saline (PBS) using the Celsee PREP100 instrument (Celsee Diagnostics) following the protocol provided by the manufacturer. The cells were then concentrated in 10-50 $\mu \mathrm{l}$ by centrifugation at $500 \mathrm{xg}$ for $10 \mathrm{~min}$, and counted using a hemocytometer. The retrieval efficiency was calculated as the percentage of the retrieved cells of the total spiked-in cells.

Retrieval of spiked-in PC3 and LNCaP cells in normal donor blood samples. A different amount of $\mathrm{PC} 3$ and $\mathrm{LNCaP}$ cells were spiked into $4 \mathrm{ml}$ of blood from healthy donors. After removal of the CD45-positive cells and enrichment of the spiked-in CTCs as aforementioned, cells enriched in the microfluidic chip were retrieved in $2 \mathrm{ml}$ PBS using the Celsee PREP100 instrument (Celsee Diagnostics) following the manufacturer's protocol. These cells were then collected by centrifugation at $500 \mathrm{x}$ g for $10 \mathrm{~min}$ and stored at $-20^{\circ} \mathrm{C}$ for future analysis by PCR amplicon sequencing.

Capturing and retrieval of CTCs in clinical blood samples. An aliquot of $4 \mathrm{ml}$ of blood sample was used to capture or retrieve CTCs using the Celsee PREP100 instrument (Celsee Diagnostics) following the protocol provided by the manufacturer. CTCs were monitored using an inverted fluorescence microscope. CTC enumeration following antibody labeling was performed manually. $\mathrm{PanCK}^{+} / \mathrm{CD}^{-} 5^{-}$nucleated cells were identified as CTCs. Positive and negative controls for antibody performance and staining were included in each experiment. After removal of CD45-positive cells and enrichment of the spiked-in CTCs as aforementioned, cells enriched in the microfluidic chip were retrieved in $2 \mathrm{ml}$ PBS using the Celsee PREP100 instrument (Celsee Diagnostics) following the manufacturer's protocol. These cells were then collected by centrifugation at $500 \mathrm{x} \mathrm{g}$ for $10 \mathrm{~min}$ and stored at $-20^{\circ} \mathrm{C}$ for future analysis by PCR amplicon sequencing. 
Table I. Primer sequences used for amplification of fragments containing mutations of interest.

\begin{tabular}{lll}
\hline Mutation & \multicolumn{1}{c}{ p.K139fs*3 } & p.T877A \\
\hline Forward outer primer & CTGAGTGACAGAGCAAGACCCTAT & AAAATCAGAGGTTGGGGAAGA \\
Reverse outer primer & AGTGTTTCTGTCATCCAAATACTCC & ACAACTTGACACTGGGCCATA \\
Forward inner primer & GTTTCTTTGCTGCCGTCTTC & CACCTCCTTGTCAACCCTGT \\
Reverse inner primer & ACACGCAAATTTCCTTCCAC & TGGGAAGCAAAGTCTGAAGG
\end{tabular}

Table II. Primer sequences used for amplification of fragments containing mutations of interest in clinical blood samples.

\begin{tabular}{ll}
\hline Mutation & \multicolumn{1}{c}{ p.T877A } \\
\hline Forward outer primer & AAAATCAGAGGTTGGGGAAGA \\
Reverse outer primer & ACAACTTGACACTGGGCCATA \\
Forward inner primer & CACCTCCTTGTCAACCCTGT \\
Reverse inner primer & TGGGAAGCAAAGTCTGAAGG \\
Forward inner primer & AGATTGCGAGAGAGCTGCAT \\
Reverse inner primer & TGCCATGGGAGGGTTAGATA \\
\hline
\end{tabular}

PCR amplicon sequencing. Mutations p.K139fs*3 of TP53 in PC3 and p.T877A of AR in LNCaP cells have been previously reported $(15,16)$. To detect these mutations, nested PCR was employed. Tables I and II lists outer and inner primer sets designed to amplify each of the mutations. The retrieved

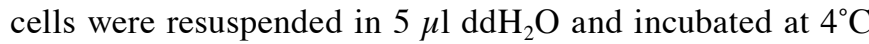
for $10 \mathrm{~min}$, and used as the template for PCR amplification. The PCR assay was set up in a $20-\mu 1$ reaction containing $10 \mu 1$ KAPA HiFi HotStart Ready Mix (Kapa Biosystems, Inc., Wilmington, MA, USA), $300 \mathrm{nM}$ of each outer primer and $1 \mu 1$ dimethyl sulfoxide (DMSO) at the following cycling conditions: $2 \mathrm{~min}$ at $95^{\circ} \mathrm{C}$, followed in turn by 3 cycles of $20 \mathrm{sec}$ at $98^{\circ} \mathrm{C}, 20 \mathrm{sec}$ at $64^{\circ} \mathrm{C}$ and $30 \mathrm{sec}$ at $72^{\circ} \mathrm{C}, 3$ cycles of $20 \mathrm{sec}$ at $98^{\circ} \mathrm{C}, 20 \mathrm{sec}$ at $61^{\circ} \mathrm{C}$ and $30 \mathrm{sec}$ at $72^{\circ} \mathrm{C}, 3$ cycles of $20 \mathrm{sec}$ at $98^{\circ} \mathrm{C}, 20 \mathrm{sec}$ at $58^{\circ} \mathrm{C}$ and $30 \mathrm{sec}$ at $72^{\circ} \mathrm{C}, 35$ cycles of $20 \mathrm{sec}$ at $98^{\circ} \mathrm{C}, 20 \mathrm{sec}$ at $57^{\circ} \mathrm{C}$ and $30 \mathrm{sec}$ at $72^{\circ} \mathrm{C}$ and $10 \mathrm{~min}$ at $72^{\circ} \mathrm{C}$. One microliter of amplified PCR products with the outer primer set was used as the template for the PCR reaction using the inner primer set under the same conditions. The final PCR products were examined on $1 \%$ agarose gel and subjected to Sanger sequencing using the inner primers after purification.

\section{Results}

Mutation detection in PC3 and LNCaP cell lines. PC3 and LNCaP cell lines are established prostate cancer cells and have been widely used in studies on prostate cancer. It has been reported that PC3 cells possess a deletion in the TP53 gene, p.K139fs*3 and LNCaP possess a missense A to $\mathrm{G}$ mutation in the AR gene, p.T887A mutation $(15,16)$. To detect these mutations, we designed primers (Table I) targeting the mutations, performed nested PCR and subsequently sequenced the amplicons by Sanger sequencing. To detect the p.T877A mutation

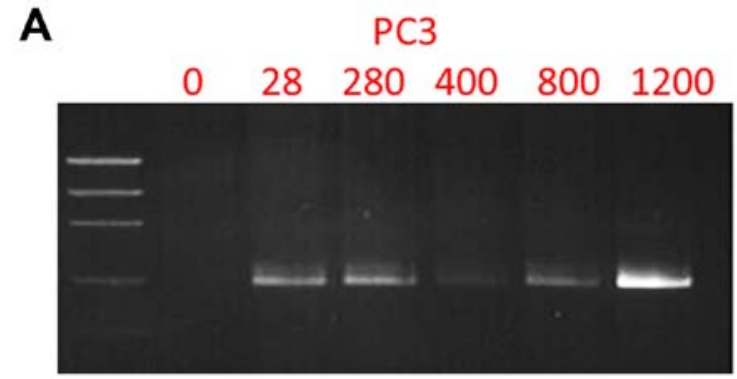

B

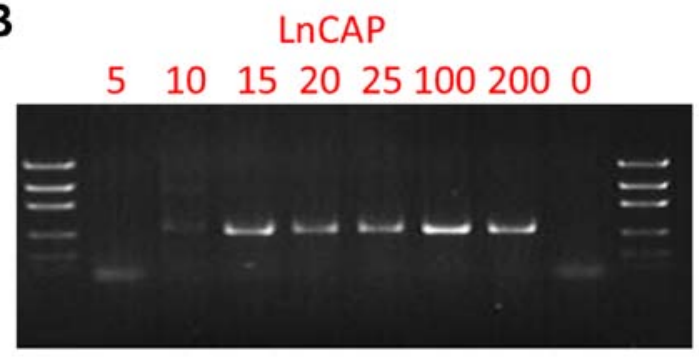

Figure 1. Nested PCR amplification of the TP53 gene from PC3 cells and the androgen receptor gene from LNCaP cells. (A) Electrophoresis gel imaging of nested PCR amplicons from 0, 28, 280, 400, 800 and 1,200 PC3 cells. (B) Electrophoresis gel imaging of nested PCR amplicons from 0, 5, 10, 20, 25, 100 and 200 LNCaP cells.

of the AR gene in the blood samples of patients, a second set of inner primers were used to improve the sensitivity of the assay (Table II). Fig. 1 reveals the PCR products and Fig. 2 reveals the Sanger sequencing results. The p.K139fs*3 and p.T887A mutations were successfully detected from 28-1,200 PC3 cells, and 10-200 LNCaP cells, respectively.

Cell retrieval efficiency using Celsee PREP100. We next tested CTC enrichment efficiency of the Celsee PREP100 by inputting a total of $110,220,330,440,550,880$ and 1,100 PC3 cells, and 60, 300, 600, 900, 1,200 and 1,500 LNCaP cells, respectively. The results are shown in Table III. Overall, the efficiency for cell retrieval was $\sim 70 \%$ with a range from 40 to $121 \%$ for PC3 and LNCaP cells. The observation that some of the cell recovery rates were $>100 \%$ was due to the variation of cell counts by hemocytometer at low cell numbers.

CTC enrichment and retrieval efficiencies using spiked-in cells in normal donor blood samples. To test the enrichment and retrieval efficiencies of CTC in blood samples, we spiked PC3 and LNCaP into $4 \mathrm{ml}$ of whole blood samples from healthy donors and stained the enriched cells for cytokeratins, CD45 and nuclei. Fig. 3 reveals typical images of 
A

TP53

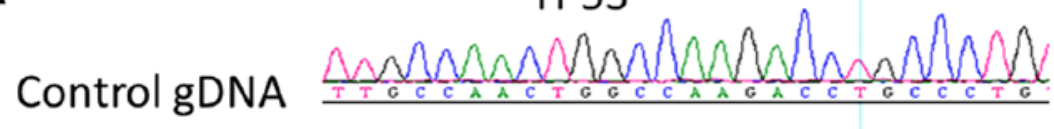

PC3 cells

B

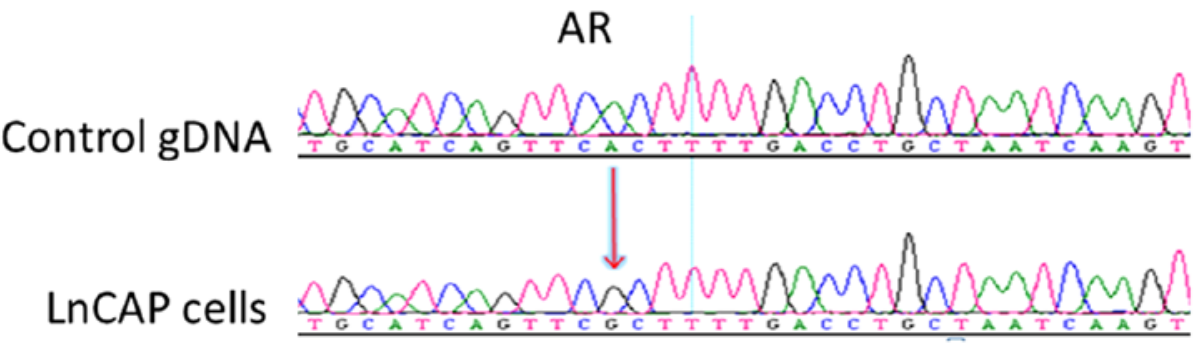

Figure 2. Sanger sequencing results of the nested PCR amplicons from PC3 and LNCaP cells. (A) Sequencing results of TP53 from control gDNA (upper panel) and PC 3 cells (lower panel), revealing a deletion in TP53 from PC3 cells. (B) Sequencing results of AR from control gDNA (upper panel) and LNCaP cells (lower panel), revealing a missense mutation in AR from LNCaP cells.

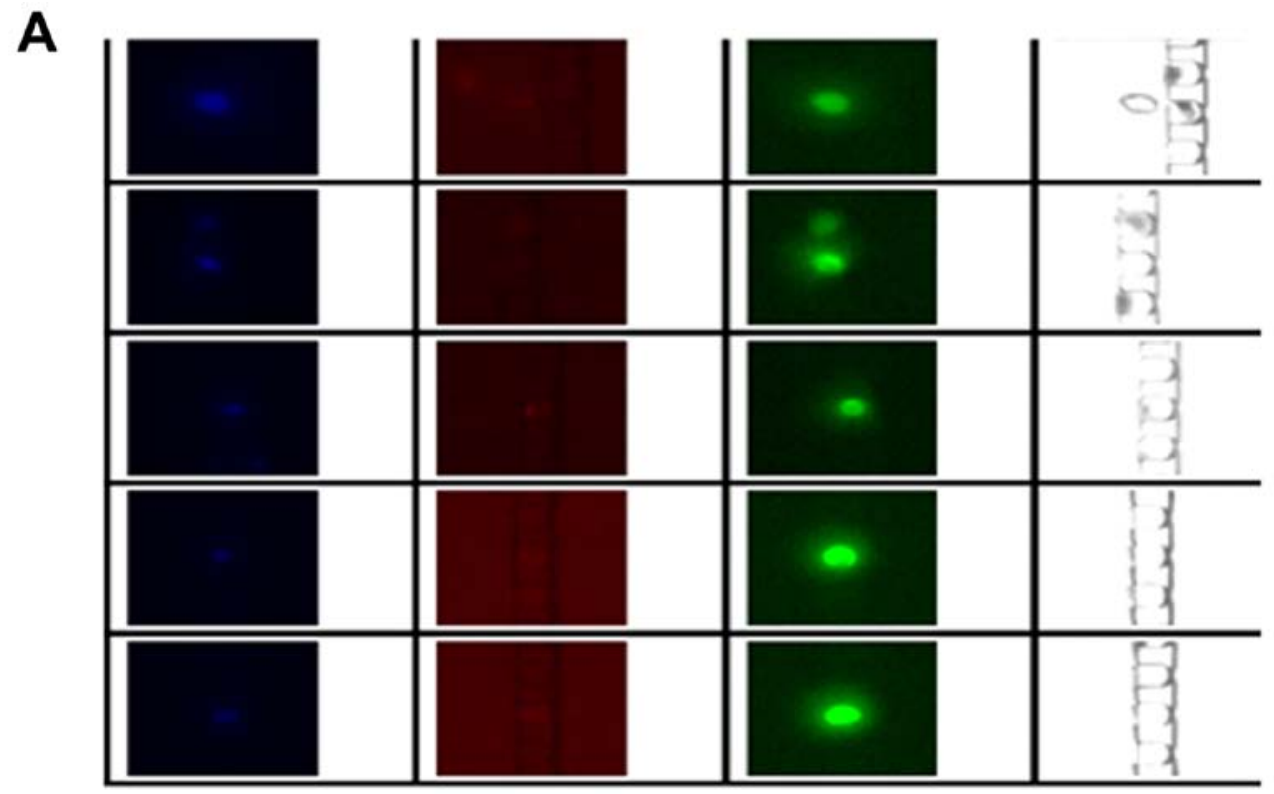

B

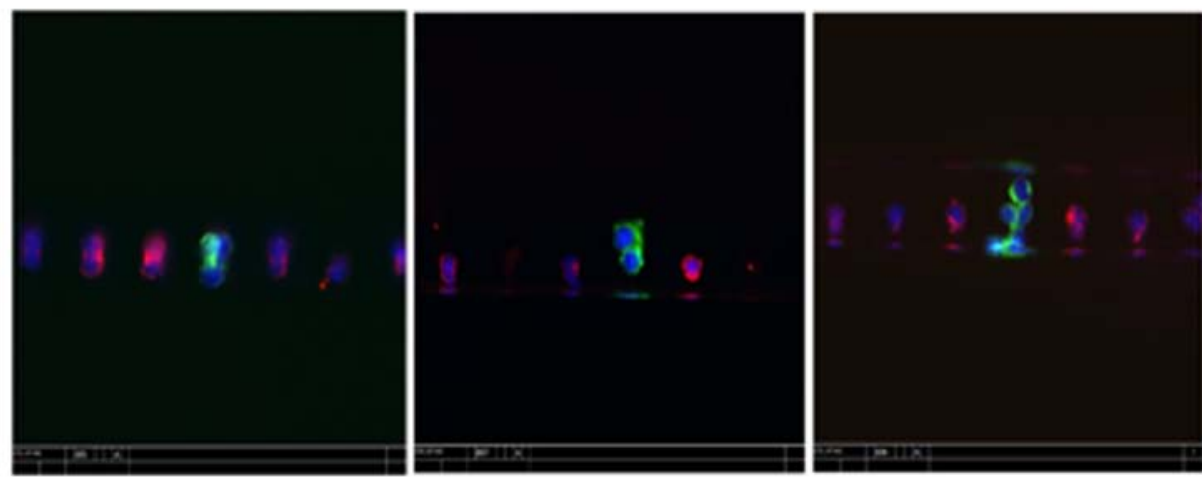

Figure 3. Representative images of the enriched cells using Celsee PREP100. Green, red and blue signals indicate cytokeratins and DAPI-positive, CD45-negative and nuclei, respectively. (A) Images from individual channels. (B) Composite images from all 3 channels revealing differential staining of CTCs (green) and leukocytes (red). It also revealed that circulating tumor cells (CTCs) are captured as a single cell, double cells and cell clusters. 
Table III. Efficiency of CTC retrieval using Celsee PREP100.

\begin{tabular}{lrrrrrr}
\hline Total input PC3 (no. of cells) & 110 & 220 & 330 & 440 & 550 & 880 \\
Retrieved PC3 (no. of cells) & 128 & 150 & 208 & 531 & 360 & 368 \\
Recovery rate (\%) & 116 & 68 & 63 & 121 & 65 & 42 \\
Total input LNCaP (no. of cells) & 60 & 300 & 600 & 900 & 1,200 & 1,200 \\
Retrieved LNCaP (no. of cells) & 58 & 250 & 618 & 605 & 1,233 & 810 \\
Recovery rate (\%) & 96 & 83 & 103 & 67 & 103 & 68 \\
\hline
\end{tabular}

CTCs, circulating tumor cells.

Table IV. Efficiency of CTC enrichment from blood samples using Celsee PREP100.

\begin{tabular}{lcc}
\hline Cell types & PC3 & LNCaP \\
\hline No. of spiked-in CTC & 250 & 50 \\
No. of total captured cells & 240 & 146 \\
No. of captured CTC cells & 96 & 37 \\
No. of background cells & 144 & 109 \\
CTC recovery rate (\%) & 38 & 74 \\
CTCs vs. background cells (ratio) & $1: 1.50$ & $1: 2.90$ \\
\hline
\end{tabular}

CTCs, circulating tumor cells.

the enriched cells in the microfluidic chip, where cytokeratins and DAPI-positive (green and blue) and CD45-negative cells were considered as CTC cells. The results (Table IV) reveals that $\sim 40 \%$ of 250 spiked-in PC3 cells and $74 \%$ of 50 spiked-in LNCaP cells were retrieved after enrichment. Furthermore, the enriched PC3 and LNCaP cells accounted for $\sim 40$ and $25 \%$ of the total retrieved cells and the ratio of captured CTCs to the background cells reached 1:1.5 for PC3 and 1:2.9 for LNCaP cells, suggesting that the removal of blood cells by the Celsee PREP100 was nearly complete and the level of remaining leukocytes in the enriched sample was very low.

CTC enrichment and retrieval using clinical blood samples. To test the enrichment and retrieval efficiencies of CTC the blood samples of patients, we stained the enriched cells for cytokeratins, CD45 and nuclei. Fig. 3 reveals typical images of the enriched cells in the microfluidic chip, where cytokeratins and DAPI-positive (green and blue) and CD45-negative cells were considered as CTC cells. For the patient samples that had CTCs, we processed another $4 \mathrm{ml}$ of the blood samples and retrieved CTCs for subsequent mutation analysis.

Mutation analysis. In order to test whether or not the mutations p.K139fs*3 of TP53 in PC3 and p.T877A of AR in LNCaP cells could be detected in the enriched cells from blood samples, we spiked 50, 100, 300 and 1,000 PC3 cells, and $25,50,100$ and $250 \mathrm{LNCaP}$ cells into $4 \mathrm{ml}$ of blood samples, depleted CD45-positive cells, enriched and retrieved the cancer cells using Celsee PREP100. The cells were then
A

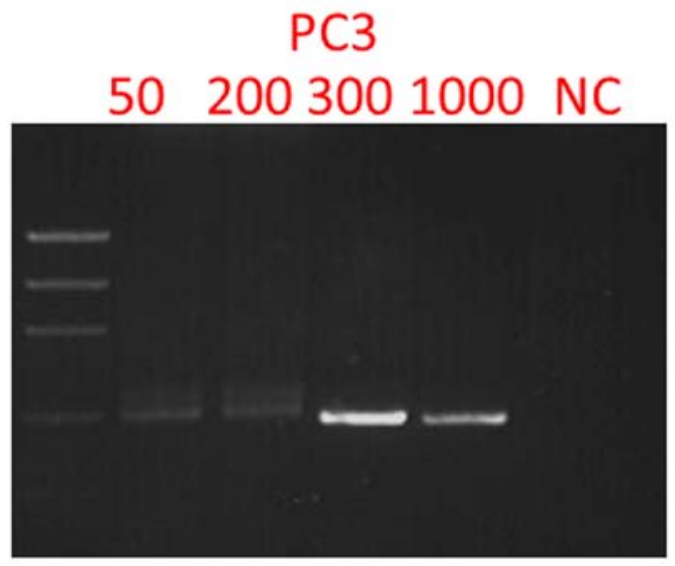

B

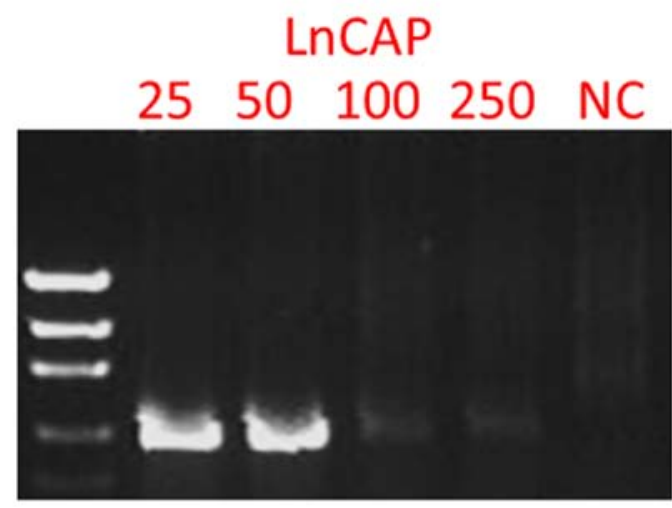

Figure 4. PCR products of the TP53 gene from the enriched PC3 cells from blood and the androgen receptor gene from enriched LNCaP cells from blood. (A) Electrophoresis gel imaging of nested PCR amplicons of the enriched cells from blood samples with spiked-in 50, 200, 300, 1,000 PC3 cells. (B) Electrophoresis of nested PCR amplicons of the enriched cells from blood samples with spiked-in 25, 50, 100 and $250 \mathrm{LNCaP}$ cells.

spun down, resuspended in water and used as templates for PCR amplification and subsequent Sanger sequencing. Fig. 4 reveals the PCR products. Fig. 5 shows the Sanger sequencing results of the PCR amplicons, indicating that both mutations could be successfully detected in the enriched cells. For the CTCs retrieved from 14 clinical blood samples, we successfully performed the mutation analysis of p.T877A. The AR mutation (heterozygous mutation) was identified in the 1 sample (CTC 37) tested (Fig. 6) and all other samples were negative for this mutation. Sixty-three CTCs were identified in sample CTC 37. 
A
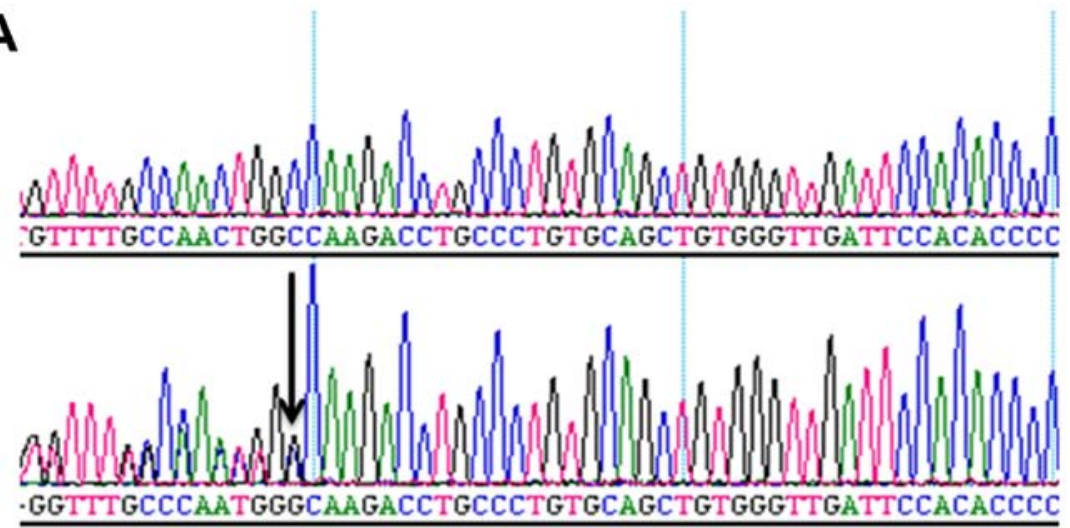

B

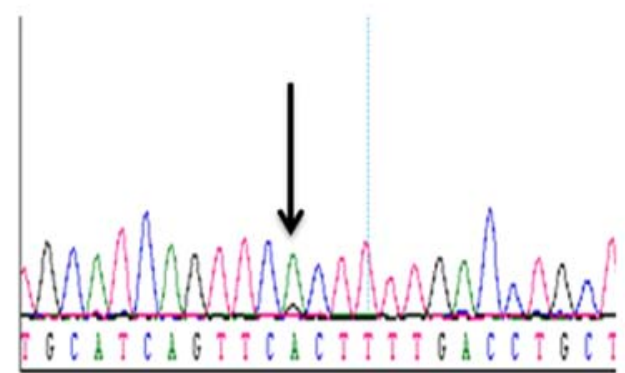

Figure 5. Sanger sequencing results of PCR amplicons of the TP53 and androgen receptor genes of the enriched PC3 and LNCaP cells from blood. (A) The upper panel is the sequencing result from the control gDNA and the lower panel is the representative sequencing result of nested PCR amplicons of the enriched PC3 cells. (B) The representative Sanger sequencing result of nested PCR amplicons of the enriched LNCaP cells. Arrows indicate the mutations.

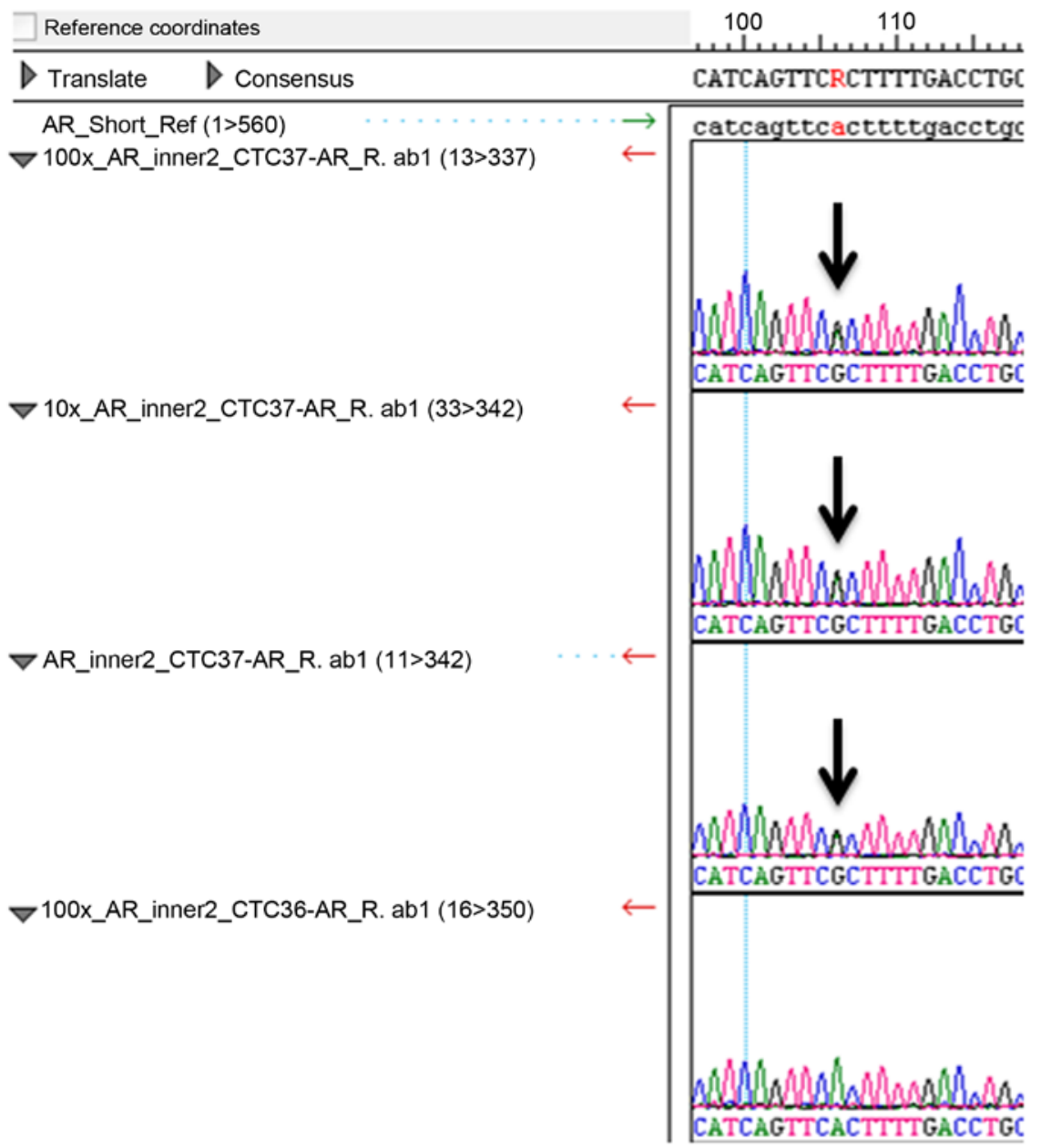

Figure 6. Sanger sequencing results of PCR amplicons of the androgen receptor gene of the enriched patient blood samples. The panel is the representative sequencing result of nested PCR amplicons of the patient blood samples (CTC 31 to CTC 41). Sample (CTC 37) carries a heterozygous AR mutation in its circulating tumor cells (CTCs). 


\section{Discussion}

Molecular characterization of CTCs has been hindered by low sensitivity and a high level of background leukocytes of currently available CTC enrichment technologies. We demonstrated that CTCs can be readily captured and further characterized with molecular markers using a simple device, Celsee PREP100. The protocol we report in the present study enriches and retrieves CTCs from blood samples based on the fact that tumor cells are larger and less deformable than normal blood cells. To evaluate the performance on cell enrichment and retrieval, we prepared prostate cancer cell lines PC3 and LNCaP and analyzed the captured cells by PCR amplicon sequencing. We were able to recover an average of $79 \%$ (ranging from 40 to $100 \%$ ) of 110-1,100 PC3 cells, and 60-1,500 LNCaP cells and detect the p.K139fs*3 deletion of TP53 in PC3 cells and the p.T877A mutation of AR in LNCaP cells. We also tested these two types of cells spiked into normal donor blood samples, captured and retrieved the cells and analyzed the retrieved cells by PCR amplicon sequencing. We were able to capture $\sim 40 \%$ of PC3 cells and $74 \%$ of $\mathrm{LNCaP}$ cells with the ratio of captured CTCs to the background leukocytes reaching 1:1.5 for PC3 and 1:2.9 for LNCaP cells. The p.K139fs*3 deletion and the p.T877A mutation were detected in the captured spiked-in PC3 and LNCaP cells, respectively. The method was also tested successfully in clinical blood samples from patients with metastatic prostate cancer. Our results demonstrated the potential of CTC molecular characterization for the diagnosis, prognosis and treatment selection of patients with metastatic malignancy.

The unique design of the microfluidic chip and Celsee PREP100 allows separation of CTCs from the background leukocytes and retrieval of the captured CTCs in a simple fashion. The variability observed on the recovery rate of cell retrieval is mainly due to the nature of the manual operation of the protocol. For example, the pressure and speed of manual pausing and pumping to retrieve the captured cells could vary from experiment to experiment and operator to operator. The variable number of background leukocytes could come from different healthy donors of the blood samples. To improve the purity of captured cells and the consistency of the protocol performance, we are developing an automated pump that can be connected to the Celsee PREP100 to retrieve captured cells from the microfluidic chip for PCR and sequencing analyses.

Enrichment of circulating cells can enable a number of downstream molecular applications. In addition to Sanger sequencing analysis used in our study, RT-PCR and DNA array assays on gene expression profiling, as well as NGS analysis on several DNA and RNA based genomic applications have been explored with enriched CTCs. Fluorescence in situ hybridization (FISH) assay on CTCs has also been demonstrated for determining gene amplification and aberrant copy number changes in cancer cells. Molecular profiling of CTCs could produce insightful information towards understanding the heterogeneity and the complexity of cancer and shed further light on the mechanisms of tumor metastasis, thus delineating tumor cells that are relevant to prognosis and therapy choice. With improvements on automation and standardization, enrichment and characterization of CTCs could overcome the technical limitations of low sensitivity and high background leukocytes and become a routine diagnostic tool in clinical use.

\section{Acknowledgements}

The authors thank all the supporting staff in the hematology/ oncology clinic, Henry Ford Health System for their support in collection of the patient blood samples.

\section{Funding}

The present study was supported in part by the pilot project funding from the Henry Ford Health System Research Administration.

\section{Availability of data and materials}

The datasets used during the present study are available from the corresponding author upon reasonable request.

\section{Authors' contributions}

RK, CH, NP, CL and YW conceived and designed the study. RK, JZ, PG, SC and WC performed the experiments. $\mathrm{CH}$ recruited and obtained consent from patients. RK, NP, CL and YW wrote the manuscript. RK, NP, CL and YW reviewed and edited the manuscript. All authors read and approved the manuscript and agree to be accounTable for all aspects of the research in ensuring that the accuracy or integrity of any part of the work are appropriately investigated and resolved.

\section{Ethics approval and consent to participate}

All experimental protocols were approved by the Institutional Review Board of the Henry Ford Health System.

\section{Consent for publication}

Not applicable.

\section{Competing interests}

The authors decalre that they have no competing interests.

\section{References}

1. Pantel $\mathrm{K}$ and Alix-Panabières C: Circulating tumor cells in cancer patients: Challenges and perspectives. Trends Mol Med 16: 398-406, 2010.

2. Maheswaran S and Haber DA: Circulating tumor cells: A window into cancer biology and metastasis. Curr Opin Genet Dev 20: 96-99, 2010.

3. Cristofanilli M, Budd GT, Ellis MJ, Stopeck A, Matera J, Miller MC, Reuben JM, Doyle GV, Allard WJ, Terstappen LW, et al: Circulating tumor cells, disease progression, and survival in metastatic breast cancer. N Engl J Med 351: 781-791, 2004.

4. Punnoose EA, Atwal SK, Spoerke JM, Savage H, Pandita A, Yeh RF, Pirzkall A, Fine BM, Amler LC, Chen DS and Lackner MR: Molecular biomarker analyses using circulating tumor cells. PLoS One 5: e12571, 2010. 
5. Allard WJ, Matera J, Miller MC, Repollet M, Connelly MC, Rao C, Tibbe AG, Uhr JW and Terstappen LW: Tumor cells circulate in the peripheral blood of all major carcinomas but not in healthy subjects or patients with nonmalignant diseases. Clin Cancer Res 10: 6897-6904, 2004.

6. Riethdorf S, Fritsche, H Müller V, Rau T, Schindlbeck C, Rack B, Janni W, Coith C, Beck K, Jänicke F, et al: Detection of circulating tumor cells in peripheral blood of patients with metastatic breast cancer: A validation study of the CellSearch system. Clin Cancer Res 13: 920-928, 2007.

7. Nagrath S, Sequist LV,Maheswaran S, Bell DW, Irimia D, Ulkus L, Smith MR, Kwak EL, Digumarthy S, Muzikansky A, et al: Isolation of rare circulating tumour cells in cancer patients by microchip technology. Nature 450: 1235-1239, 2007.

8. Khoo BL, Warkiani ME, Tan DS, Bhagat AA, Irwin D, Lau DP Lim AS, Lim KH, Krisna SS, Lim WT, et al: Clinical validation of an ultra high-throughput spiral microfluidics for the detection and enrichment of viable circulating tumor cells. PLoS One 9: e99409, 2014.

9. Stott SL, Hsu CH, Tsukrov DI, Yu M, Miyamoto DT, Waltman BA, Rothenberg SM, Shah AM, Smas ME, Korir GK, et al: Isolation of circulating tumor cells using a microvortex-generating herringbone-chip. Proc Natl Acad Sci USA 107: 18392-18397, 2010.

10. Saliba AE, Saias L, Psychari E, Minc N, Simon D, Bidard FC, Mathiot C, Pierga JY, Fraisier V, Salamero J, et al: Microfluidic sorting and multimodal typing of cancer cells in self-assembled magnetic arrays. Proc Natl Acad Sci USA 107: 14524-14529, 2010.

11. Powell AA, Talasaz AH, Zhang H, Coram MA, Reddy A, Deng G, Telli ML, Advani RH, Carlson RW, Mollick JA, et al Single cell profiling of circulating tumor cells: Transcriptional heterogeneity and diversity from breast cancer cell lines. PLoS One 7: e33788, 2012.
12. Sieuwerts AM, Kraan J, Bolt-de Vries J, van der Spoel P, Mostert B, Martens JW, Gratama JW, Sleijfer S and Foekens JA: Molecular characterization of circulating tumor cells in large quantities of contaminating leukocytes by a multiplex real-time PCR. Breast Cancer Res Treat 118: 455-468, 2009.

13. Riahi R, Gogoi P, Sepehri S, Zhou Y, Handique K, Godsey J and Wang Y: A novel microchannel-based device to capture and analyze circulating tumor cells (CTCs) of breast cancer. Int J Oncol 44: 1870-1878, 2014.

14. Gogoi P, Sepehri S, Zhou Y, Gorin MA, Paolillo C, Capoluongo E, Gleason K, Payne A, Boniface B, Cristofanilli M, et al: Development of an automated and sensitive microfluidic device for capturing and characterizing circulating tumor cells (CTCs) from clinical blood samples. PLoS One 11: e0147400, 2016.

15. Chang H, Jackson DG, Kayne PS, Ross-Macdonald PB, Ryseck RP and Siemers NO: Exome sequencing reveals comprehensive genomic alterations across eight cancer cell lines. PLoS One 6: e21097, 2011.

16. Veldscholte J, Ris-Stalpers C, Kuiper GG, Jenster G, Berrevoets C, Claassen E, van Rooij HC, Trapman J, Brinkmann AO and Mulder E: A mutation in the ligand binding domain of the androgen receptor of human LNCaP cells affects steroid binding characteristics and response to anti-androgens. Biochem Biophy Res Commun 173: 534-540, 1990.

This work is licensed under a Creative Commons Attribution-NonCommercial-NoDerivatives 4.0 International (CC BY-NC-ND 4.0) License. 